\title{
The Enthalpies of Combustion and Formation of 2,2'-Dichloroethyl Sulfide
}

\author{
Walter H. Johnson \\ Institute for Materials Research, National Bureau of Standards, Washington, D.C. 20234
}

(June 10, 1975)

\begin{abstract}
The enthalpies of combustion and formation of $2,2^{\prime}$-dichloroethyl sulfide (mustard gas) have been determined by combustion in an adiabatic rotating-bomb calorimeter. The bomb process has been corrected to:$$
\mathrm{C}_{4} \mathrm{H}_{8} \mathrm{Cl}_{2} \mathrm{~S}(\text { liq })+7 \mathrm{O}_{2}(\mathrm{~g})+98 \mathrm{H}_{2} \mathrm{O}(\text { liq }) \rightarrow 4 \mathrm{CO}_{2}(\mathrm{~g})+\left[2 \mathrm{HCl}+\mathrm{H}_{2} \mathrm{SO}_{4}+100 \mathrm{H}_{2} \mathrm{O}\right](\mathrm{liq})
$$

for which the following values were obtained:

$$
\begin{aligned}
& \Delta H c^{\circ}\left(25^{\circ} \mathrm{C}\right)=-3163.49 \pm 1.26 \mathrm{~kJ} / \mathrm{mol} \text { and } \\
& \Delta H f^{\circ}\left(25^{\circ} \mathrm{C}\right)=-200.57 \pm 1.58 \mathrm{~kJ} / \mathrm{mol} \text {. }
\end{aligned}
$$

Key words: Combustion; dichloroethyl sulfide; enthalpy; formation; heat; mustard gas.

\section{Introduction}

The enthalpy of formation of $2,2^{\prime}$-dichloroethyl sulfide (more commonly known as mustard gas) has been determined by combustion of the liquid material in an adiabatic rotating-bomb calorimeter. This work was undertaken for Edgewood Arsenal under contract A133051801F4W9 to devise a reliable procedure for the combustion calorimetry of compounds containing both chlorine and sulfur. The problems associated with the combustion of chlorine compounds and those associated with the combustion of sulfur compounds are combined when the compound contains both of these elements. There appears to be no data available on the combustion calorimetry of compounds of this type in the literature. The compound 2,2' -dichloroethyl sulfide was selected because (1) there was need for reliable data on the enthalpies of combustion and formation for this substance and (2) we were able to obtain the results of an unpublished prior investigation [1]. ${ }^{1}$ The data consisted of the results of two combustion experiments, performed in 1918, in which the nature and quantities of the combustion products were not well-defined. Because of the corrosive nature and the relatively high heats of solution and dilution of the products of combustion, a platinum-lined bomb and a rotating-bomb calorimeter are required.

Because the main purpose of this investigation was to develop calorimetric procedures for compounds containing both chlorine and sulfur, no particular emphasis was placed on the use of a well characterized sample.

${ }^{1}$ Figures in brackets indicate the literature references at the end of this paper
However, since no previous data on compounds of this type are available, it was decided to publish the results of this investigation.

\section{Materials}

\subsection{2,2'-Dichloroethyl Sulfide}

The sample was obtained from $\mathrm{K}$ and $\mathrm{K}$ Laboratories, Inc., Plainview, N.Y. The sample had a pale amber color which was taken to indicate a significant amount of impurity. The material was distilled in vacuum; about 10 percent of the sample was discarded. The distillate was a clear liquid for which the freezing point was $13.4 \pm 0.2{ }^{\circ} \mathrm{C}$.

Analyses of the products of combustion yielded the following results in weight percent:

$\begin{array}{lcr} & \text { Found } & \text { Theor. } \\ \text { C } & 30.21 & 30.20 \\ \text { S } & 20.11 & 20.15 \\ \text { Cl } & 44.29 & 44.57\end{array}$

The quantity of chloroplatinic acid, usually formed when chlorine compounds are burned in the presence of platinum, was not determined but would be expected to account for approximately 0.3 percent of the chlorine.

\subsection{Cotton Fuse}

The fuse was taken from a spool of cotton thread which was determined to have the empirical formula $\mathrm{CH}_{1.730} \mathrm{O}_{0.853}$. The density of the thread was $0.87 \mathrm{~g} / \mathrm{cm}^{3}$. 
The energy of combustion, determined in a separate experiment, gave $\Delta U c^{\circ}\left(28^{\circ} \mathrm{C}\right)=-17621 \mathrm{~J} / \mathrm{g}$.

\subsection{Arsenious Oxide Solution}

The arsenious oxide solution was prepared by dissolving arsenic trioxide, SRM 83B, in water. The concentration of the solution was determined by potentiometric titration with standard potassium permanganate. The concentration of the arsenious oxide solution was $23.345 \mu \mathrm{mol} / \mathrm{cm}^{3}$.

\subsection{Oxygen}

High purity oxygen was used for both calibration and combustion experiments. An analysis furnished with the cylinder gave the following impurities in ppm; $\mathrm{Kr}, 12.0 ; \mathrm{Xe}, 0.7 ; \mathrm{N}_{2} \mathrm{O}, 0.8 ; \mathrm{N}_{2}, 3.0$; and $\mathrm{H}_{2} \mathrm{O}, 3.0$. The presence of these impurities is expected to have no significant effect on the experimental values obtained for the combustion experiments.

\subsection{Nitrogen}

A cylinder of water-pumped nitrogen was used. This nitrogen was essentially free of combustibles and carbon dioxide but contained some water; for the relatively small quantity of nitrogen that was used this amount of water is negligible.

\section{Apparatus and Procedure}

The adiabatic rotating-bomb calorimeter, the automatic shield-temperature controls and the temperaturemeasuring system employed in the investigation have been described [2].

A sample of the liquid material was weighed into a platinum crucible and placed in the crucible support of the platinum-lined silver bomb. A glass cup containing $4.000 \mathrm{~cm}^{3}$ of arsenious oxide solution was placed on the bottom of the bomb cavity. About 1.5 $\mathrm{cm}$ of cotton string was attached to $1 \mathrm{~cm}$ of platinum wire, $0.075 \mathrm{~mm}$ in diameter, which was connected between the electrodes. The cotton string was positioned such that the lower end was in contact with the sample. The bomb was then sealed and $2.34 \mathrm{~atm}^{2}$ of nitrogen was introduced; oxygen was then added to bring the total pressure up to 31.62 atm.

The bomb was transferred to the adiabatic calorimeter, the temperature of filling was measured and the bomb was heated electrically to $24.99^{\circ} \mathrm{C}$. The calorimeter jacket was evacuated, the adiabatic temperature controls were placed in operation and the system was left under control overnight to reach temperature equilibrium.

On the following morning temperatures were observed at intervals of a few minutes during an initial rating period and the sample was ignited by discharging an $18000 \mu \mathrm{F}$ capacitor previously charged to $24 \mathrm{~V}$, through the platinum wire fuse. The platinum wire ignited the cotton string which in turn ignited the

${ }^{2} 1$ atmosphere $=101.325 \mathrm{kPa}$ sample. After two minutes the bomb was rotated to mix the contents of the bomb solution. When thermal equilibrium was established, temperatures were observed during a final rating period; the resulting time-temperature curves were extrapolated to the time of firing to obtain the initial and final calorimeter temperatures

The bomb was removed from the calorimeter, connected to a gas train and the gaseous contents were passed successively through a drying tube containing magnesium perchlorate and phosphorus pentoxide, and through a weighed absorption tube containing Ascarite, ${ }^{3}$ magnesium perchlorate and phosphorus pentoxide. In each case, the absorption tube was flushed with dry hydrogen before weighing and after the absorption of carbon dioxide to reduce the correction for displacement of gas by the sodium carbonate; a tare was used to reduce the effect of changes in humidity and air density between weighings. The quantity of carbon dioxide was determined by the increase in weight of the absorption tube.

The bomb was opened and the bomb solution transferred to a weighed polyethylene bottle. A weighed aliquot of the bomb solution was titrated potentiometrically with standard potassium permanganate solution using a microburet. This determination is very precise and the end point can be determined within \pm 0.001 $\mathrm{cm}^{3}$ of $0.1 N$ soln. A similar titration of the initial solution compared with the bomb solution gives an accurate measure of the remaining $\mathrm{As}^{+++}$. Another aliquot was taken and analyzed for nitric acid by means of a modified Kjeldahl method [3]. The modifications involved heating of the reducing mixture with an infrared lamp instead of an electric heating element in the solution.

When chlorine compounds are burned about 80 percent of the chlorine forms hydrogen chloride and the remainder goes to gaseous chlorine. To insure the complete conversion to hydrochloric acid an excess of a reducing agent such as arsenious oxide is placed in the bomb. Also, in the combustion of sulfur compounds a significant part of the sulfur is found as sulfur dioxide. However, when a small amount of nitrogen is present in the bomb atmosphere the sulfur is all converted to sulfur trioxide which dissolves in the solution forming sulfuric acid. The general procedure for sulfur-containing compounds is to leave one atmosphere of air in the bomb before filling with oxygen. In this investigation, however, the conversion to sulfuric acid was found to be very slow, involving several hours for complete conversion. When at least two atmospheres of nitrogen was used the conversion was rapid and complete.

There was also the possibility that there would be some oxidation of the arsenious acid by the sulfuric acid. Experiments on the combustion of thianthrene in the presence of arsenious acid showed no significant change in the quantity of $\mathrm{As}^{+++}$between the initial and final bomb solutions; therefore, the oxidizing

\footnotetext{
${ }^{3}$ Certain commercial materials are identified in this paper in order to specify adequately the experimental procedures. Such identification does not imply recommendation or en dorsement by the National Bureau of Standards.
} 
effect on the arsenious acid by the sulfuric acid produced under experimental conditions was considered not significant.

\section{Results and Calculations}

The results of the analyses of the combustion products is given in table 1 . The $\mathrm{CO}_{2}$-ratio was obtained by subtracting the theoretical quantity calculated from the mass of the cotton fuse from the observed total mass of carbon dioxide and dividing the result by the theoretical quantity calculated from the mass of sample.

TABLE 1. Results of the analyses of the combustion products

\begin{tabular}{l|c|c|c|c}
\hline \hline & 551 & 552 & 553 & 554 \\
\hline Mass of sample, g...... & 0.311666 & 0.242445 & 0.306338 & 0.433452 \\
Mass of cotton, g....... & .000966 & .000636 & .000739 & .000835 \\
$\mathrm{CO}_{2}-\mathrm{ratio} \ldots \ldots \ldots \ldots \ldots \ldots$ & .9970 & .9953 & .9962 & 1.0126 \\
$\mathrm{As}_{2} \mathrm{O}_{5}, \mu$ mol................. & 57.95 & 51.69 & 70.93 & 92.92 \\
$\mathrm{HNO}_{3}$ mmol ............ & 0.1186 & 0.1494 & 0.2475 & 0.3231 \\
\hline
\end{tabular}

The quantities of $\mathrm{As}_{2} \mathrm{O}_{5}$ and $\mathrm{HNO}_{3}$ were those determined by the permanganate titrations and the microKjeldahl analyses. The quantities of $\mathrm{HCl}$ and $\mathrm{H}_{2} \mathrm{SO}_{4}$ were assumed to be stoichiometric.

The following values were used in the calculations:
Benzoic acid $2,2^{\prime}$-Dichloroethyl Sulfide

Density,
$\mathrm{g} \cdot \mathrm{cm}^{-3}$
1.32

1.27 $\left(\frac{\partial U}{\partial P}\right)_{T}$ $\mathrm{J} \cdot \mathrm{g}^{-1}$. atm $^{-1}$ $-0.0126$

0
The 1971 table of atomic weights [4] were used throughout this paper. The unit of energy is the joule; for conversion from the conventional thermochemical calorie, 1 calorie $=4.184$ joules.

The enthalpy of oxidation of $\mathrm{As}_{2} \mathrm{O}_{3(\mathrm{aq})}$ to $\mathrm{As}_{2} \mathrm{O}_{5}$ (aq) was taken as $-319.82 \mathrm{~kJ} / \mathrm{mol}$ from the data of Sellers, Sunner and Wadso [5]. The heat of decomposition of aqueous nitric acid into nitrogen and water was taken as $\Delta U_{c}^{\circ}\left(28^{\circ} \mathrm{C}\right)=-59.7 \mathrm{~kJ} / \mathrm{mol}$.

The effective energy equivalent of the calorimeter was determined by means of a series of combustion experiments using benzoic acid, SRM 39i. The certificate value for the energy of combustion under certificate conditions is $-26434 \mathrm{~J} / \mathrm{g}$ at $25{ }^{\circ} \mathrm{C}$; this corresponds to $-26410.36 \mathrm{~J} / \mathrm{g}$ for the isothermal bomb process under standard conditions at $28{ }^{\circ} \mathrm{C}$. The results of the benzoic acid calibration experiments are given in table 2. EEE-std is the effective energy equivalent of the standard calorimeter which includes the empty bomb with all internal platinum parts except the crucible; $C v$-cont $(i)$ is the heat capacity of the crucible, sample, oxygen and water initially placed in the bomb; Corr to tm is a correction for deviation of the actual mean temperature of the system from the standard mean temperature of $26.5^{\circ} \mathrm{C}$; Corr-parts is a correction for the effect of changes made to the system during the series of experiments and EEEactual is the effective energy equivalent of the actual calorimeter system obtained as the sum of EEE-std and the above corrections.

Temperature rise is the observed increase in temperature of the system following ignition of the sample. Q-total is the total energy evolved by the over-all bomb process obtained as the product of EEE-actual and the temperature rise; $q$-ign is the electrical energy introduced to ignite the sample; q-decomp $\mathrm{HNO}_{3}$ is the calculated quantity of energy required to decompose the nitric acid, formed in the combustion from traces of nitrogen in the bomb atmosphere, to nitrogen, oxygen and water; $q-W C$ is the Washburn correction [6] applied to convert the initial and final contents of the bomb to their respective standard states at the actual final temperature and $q$-corr to $28{ }^{\circ} \mathrm{C}$ is a correction to convert the energy evolved by the standard reaction at the actual final temperature to the standard final temperature. Q-std reaction is the energy evolved by the isothermal bomb process at the standard final temperature, obtained as Q-total $+\Sigma q$; division by the mass of sample yields $\Delta U_{C}^{\circ}$.

The results of the benzoic acid calibration experiments were calculated by means of a computer program. For these experiments, $E E E$-std was calculated by iteration from the observed temperature rise and mass of sample taking $\Delta U_{c}^{\circ}\left(28^{\circ} \mathrm{C}\right)$ equal to -26410.36 $\mathrm{J} / \mathrm{g}$.

The results of the combustion experiments on $2,2^{\prime}-$ dichloroethyl sulfide are given in table 3 . The same format has been used but some additional corrections have been listed; $q$-comb fuse is the energy of combustion of the cotton thread used to ignite the sample; q-oxid $\mathrm{As}_{2} \mathrm{O}_{3}$ is the energy evolved by oxidation of $\mathrm{As}_{2} \mathrm{O}_{3}$ in the reduction of gaseous chlorine to hydrogen chloride; $q$-diln $\mathrm{H}_{2} \mathrm{SO}_{4}$ and $\mathrm{HCl}$ are applied to convert the actual final concentrations to the selected final concentrations of 1:100 $\mathrm{H}_{2} \mathrm{O}$ and 1:50 $\mathrm{H}_{2} \mathrm{O}$ respectively.

No computer program was available which could accept compounds containing both sulfur and chlorine, it was necessary, therefore, to compute these data manually. Because data on the solubility and heats of solution of oxygen, nitrogen, and carbon dioxide in the resulting solution of sulfuric, hydrochloric, arsenious and arsenic acids were not available it was necessary to make certain assumptions in calculation of the Washburn correction; in each case the heat of solution was taken equal to that in pure water. The error introduced by these assumptions is believed to be less than 10 percent in the correction or $\pm 0.5 \mathrm{~J}$.

The results given in table 2 correspond to the reaction:

$$
\begin{aligned}
& \mathrm{C}_{4} \mathrm{H}_{8} \mathrm{Cl}_{2} \mathrm{~S}(\mathrm{liq})+7 \mathrm{O}_{2}(\mathrm{~g})+98 \mathrm{H}_{2} \mathrm{O}(\mathrm{liq}) \rightarrow \\
& \quad 4 \mathrm{CO}_{2}(\mathrm{~g})+\left[2 \mathrm{HCl}+\mathrm{H}_{2} \mathrm{SO}_{4}+100 \mathrm{H}_{2} \mathrm{O}\right](\text { liq })
\end{aligned}
$$

$\Delta U \&\left(28^{\circ} \mathrm{C}\right)=-3156.82 \pm 1.26 \mathrm{~kJ} / \mathrm{mol}$ 
TABLE 2. Results of the benzoic acid calibration experiments

\begin{tabular}{|c|c|c|c|c|c|c|}
\hline Expt. No. & 495 & 524 & 525 & 526 & 527 & 528 \\
\hline 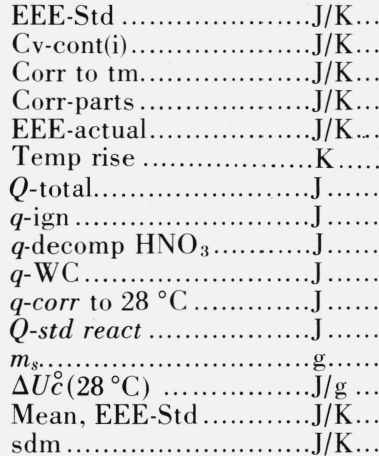 & $\begin{array}{c}2519.50 \\
4.82 \\
.08 \\
-.16 \\
2524.24 \\
3.133364 \\
-7909.36 \\
1.22 \\
.70 \\
6.39 \\
-.04 \\
-7901.10 \\
.299167 \\
-26410.36 \\
2519.56 \\
.048\end{array}$ & $\begin{array}{r}2519.67 \\
4.81 \\
.11 \\
.00 \\
2524.59 \\
3.108335 \\
-7847.29 \\
1.06 \\
.21 \\
6.32 \\
-.04 \\
-7839.73 \\
.296843 \\
-26410.36\end{array}$ & $\begin{array}{c}2519.44 \\
4.78 \\
.15 \\
.00 \\
2524.37 \\
3.159280 \\
-7975.18 \\
1.18 \\
.30 \\
6.38 \\
-.06 \\
-7967.38 \\
.301676 \\
-26410.36\end{array}$ & $\begin{array}{r}2519.66 \\
4.79 \\
.09 \\
.00 \\
2524.55 \\
3.089474 \\
-7799.53 \\
.79 \\
.47 \\
6.26 \\
-.03 \\
-7792.05 \\
.295038 \\
-26410.36\end{array}$ & $\begin{array}{r}2519.65 \\
4.76 \\
.10 \\
.00 \\
2524.51 \\
3.100061 \\
-7826.14 \\
1.11 \\
.17 \\
6.22 \\
-.04 \\
-7818.67 \\
.296046 \\
-26410.36\end{array}$ & $\begin{array}{r}2519.42 \\
4.81 \\
.14 \\
.00 \\
2524.37 \\
3.173467 \\
-8011.01 \\
1.16 \\
.84 \\
6.46 \\
-.06 \\
-8002.62 \\
.303011 \\
-26410.36\end{array}$ \\
\hline
\end{tabular}

TABLE 3. Results of the combustion experiments on $2,2^{\prime}$ dichloroethyl sulfide

\begin{tabular}{|c|c|c|c|c|}
\hline Expt. No. & 551 & 552 & 553 & 554 \\
\hline 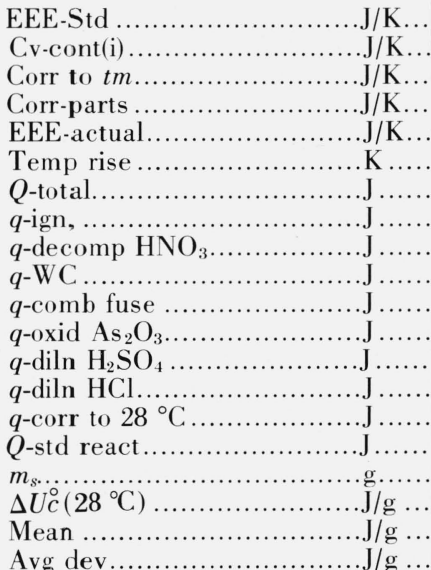 & $\begin{array}{c}2519.56 \\
28.01 \\
-0.48 \\
.00 \\
2547.09 \\
2.446197 \\
-6230.68 \\
.81 \\
7.08 \\
4.01 \\
16.67 \\
18.53 \\
.37 \\
.50 \\
-.29 \\
-6183.00 \\
.311666 \\
-19838.55 \\
-19845.05 \\
\pm 4.46\end{array}$ & $\begin{array}{c}2519.56 \\
27.92 \\
-0.97 \\
.00 \\
2546.51 \\
1.905797 \\
-4853.13 \\
.84 \\
8.92 \\
3.31 \\
10.98 \\
16.53 \\
.80 \\
.04 \\
-.46 \\
-4812.17 \\
.242445 \\
19848.50\end{array}$ & $\begin{array}{c}2519.56 \\
28.01 \\
-0.54 \\
.00 \\
2547.03 \\
2.409353 \\
-6136.69 \\
.73 \\
14.78 \\
4.12 \\
12.76 \\
22.69 \\
.42 \\
.55 \\
-.32 \\
-6080.96 \\
.306338 \\
19850.50\end{array}$ & $\begin{array}{c}2519.56 \\
28.22 \\
0.38 \\
.00 \\
2548.16 \\
3.394446 \\
-8649.59 \\
.76 \\
19.29 \\
5.65 \\
14.41 \\
29.71 \\
-.51 \\
-1.02 \\
.31 \\
-8580.99 \\
.432452 \\
19842.65\end{array}$ \\
\hline
\end{tabular}

$\Delta H \&\left(28^{\circ} \mathrm{C}\right)=-3164.33 \pm 1.26 \mathrm{~kJ} / \mathrm{mol}$

$\Delta H \varepsilon\left(25^{\circ} \mathrm{C}\right)=-3163.49 \pm 1.26 \mathrm{~kJ} / \mathrm{mol}$

$$
\begin{array}{r}
\Delta H f^{\circ}\left(25{ }^{\circ} \mathrm{C}\right)=-200.57 \pm 1.58 \mathrm{~kJ} / \mathrm{mol} \\
(-47.94 \pm 0.38 \mathrm{kcal} / \mathrm{mol})
\end{array}
$$

The uncertainty assigned to the value for $\Delta H^{\circ}$ was obtained by combining (as the square root of the sum of squares) $2 \mathrm{sdm}$ for the calibration experiments, the average deviation of the combustion experiments, 0.01 percent for the certified value of benzoic acid, 0.02 percent for purity of the sample, 0.01 percent for calculation of the $\mathrm{W}$ ashburn correction, 0.01 percent for calculation of the energy of oxidation of $\mathrm{As}_{2} \mathrm{O}_{3}$ and 0.02 percent for the uncertainty in the energy of mixing of sulfuric acid with the hydrochloric acid-arsenious acid solution. The auxiliary data were obtained by Selected Values of Chemical Thermodynamic Properties, NBS Tech. Note 270-3 [7].

The uncertainty assigned to $\Delta H f^{\circ}$ was obtained by combining as the square root of the sum of the squares the uncertainty of $\Delta H^{\circ}$ with 0.01 percent in the accepted values for the heats of formation of carbon dioxide, water, aqueous sulfuric acid and aqueous hydrochloric acid [7].

\section{Discussion}

There are no previous data on the heat of combustion of $2,2^{\prime}$-dichloroethyl sulfide in the available literature. An investigation by R. S. Hall and J. L. Hutchinson of the Chemical Laboratory, Edgewood Arsenal [1], EA-L-87 (1918) and a recalculation of their data by Leo Finkelstein [8], E. A. T. R. 259, dated March 1937 are the only values known.

The recalculation by Finkelstein, gave for the reaction:

$$
\begin{aligned}
\mathrm{C}_{4} \mathrm{H}_{8} \mathrm{Cl}_{2} \mathrm{~S}(\mathrm{l})+7.5 \mathrm{O}_{2}(\mathrm{~g})=4 \mathrm{CO}_{2}(\mathrm{~g})+4 \mathrm{H}_{2} \mathrm{O}(\mathrm{l}) \\
+\mathrm{SO}_{3}(\mathrm{c})+\mathrm{Cl}_{2}(\mathrm{~g}) \Delta H=-731.83 \mathrm{kcal} / \mathrm{mol} .
\end{aligned}
$$


Correcting for recent changes in the enthalpies of formation of the products of combustion gives -733.34 $\mathrm{kcal} / \mathrm{mol}(-3068.29 \mathrm{~kJ} / \mathrm{mol})$ for the process according to reaction (2). By combination of reaction (2) with the appropriate heats of formation and reaction, the energy of the process corresponding to reaction (1) is found to be $-3260.47 \mathrm{~kJ} / \mathrm{mol}$. The difference of 3 percent between the results of the present investigation and that obtained by Hall and Hutchinson is not surprising in view of the problems associated with their measurements. The discrepancy is obviously due to a number of factors including the reaction with the bomb surface, and the bomb gasket, the escape of gases during combustion, the nonhomogeneity of the bomb solution, and errors in the determination of the nature and quantities of the combustion products.

\section{References}

[1] Hall, R. S., and Hutchinson, J. L., Chemical Laboratory, Edge- wood Arsenal, EA-L-87 (1918) (unpublished).

[2] Prosen, E. J., and Johnson, W. H., in preparation. For a partial description see Armstrong, G. T., and Johnson, W. H., National Bureau of Standards, informal report, July 1973.

[3] Kieselbach, R., Ind. and Eng. Chem. 16, 764 (1944).

[4] Commission on Atomic Weights, IUPAC, Pure and Applied Chem. 30, 637-649 (1972); Atomic Weights of the Elements 1971.

[5] Sellers, P., Sunner, S., and Wadso, I., Acta Chem. Scand. 18, 202 (1964)

[6] Washburn, E. W., J. Res. Nat. Bur. Stand. (U.S.) 10, 525 (1933). RP546.

[7] Wagman, D. D., Evans, W. H., Parker, V. B., Halow, I., Bailey, S. M., and Schumm, R. H., Nat. Bur. Stand. (U.S.), Tech. Note 270-3, 264 pages, (Jan. 1967).

[8] Finkelstein, L., Chemical Laboratory, Edgewood Arsenal, E. A. T. R. 259 (1937) (unpublished).

(Paper 79A5-865) 